\title{
HOW SPECIES DIVERSITY COULD REDUCE THE PREVALENCE OF INFECTIOUS DISEASES
}

\author{
COLLINS, O. C. - DUFFY, K. J.* \\ Institute of Systems Science, Durban University of Technology \\ Durban 4000, South Africa \\ (phone: +27-31-373-6733) \\ *Corresponding author \\ e-mail: kevind@dut.ac.za; phone+27-31-373-6733 \\ (Received $13^{\text {th }}$ Nov 2017; accepted $27^{\text {th }}$ Feb 2018)
}

\begin{abstract}
Understanding the benefits of biodiversity conservation is essential for developing policies that can enhance the environment and hence the well-being of humans as well. We formulate a consumerresources model for a terrestrial ecosystem that incorporates resource diversity in the form of multiple species of resources (for example trees, grasses or both) and the consumers of those resources. This model is used to explore a potential benefit of maintaining ecosystem biodiversity, namely reducing the spread of infectious diseases. The important mathematical features of the model are derived, analysed and used to investigate our hypothesis that increasing species diversity in the resources can reduce diseases in those resources. The results of the analyses and numerical simulations suggest that indeed increasing resource diversity can lead to a decrease in infectious diseases. Of more interest, we show how increased diversity can result in eradication of disease in cases where disease persistence would normally be predicted.
\end{abstract}

Keywords: consumer-resource model, ecosystem epidemiology dynamics, stability analysis, bifurcation, biodiversity

\section{Introduction}

The economic and environmental benefits of biodiversity are well known (Pimentel et al., 1997). Primarily, ecosystems that are species-rich are more resilient and stabilize productivity over time (Naeem, 2009; Patz et al., 2005; Naeem and Li, 1997; Bai et al., 2004; Hautier et al., 2015). Recently, Isbell et al. (2015) showed how biodiversity increases the resistance of ecosystem productivity to a broad range of climate events.

The effects of biodiversity on human well-being are multifaceted. For example, the links between biodiversity and employment have been established Nunes et al. (2011). In health, ecosystem biodiversity plays a crucial role in regulating the transmission of many infectious diseases among human populations (WHO, 2016; Patz et al., 2005). Further evidence from the literature suggests that biodiversity conservation could promote human and wildlife health (Ostfeld and Keesing, 2012). Statistics have shown that about sixty percent of emerging infectious diseases that affect humans originate in animals and more than two-thirds of these originate in wildlife Patz et al. (2005). Meanwhile, according to the World Health Organization (WHO, 2016) zoonoses still constitute significant public health problems.

The occurrence of infectious diseases in ecosystems could be one of the factors affecting their productivity. The epidemiology of ecosystems have been investigated theoretically using a number of different approaches (Kooi et al., 2011; Auger et al., 2009; Das et al., 2011; Tewa et al., 2013) and it has been shown that maintaining biodiversity can affect disease transmission (Ezenwa et al., 2006; Haas et al., 2011; 
Johnson et al., 2013; Ostfeld and Keesing, 2012). For instance: Haas et al. (2011) demonstrated that forest species diversity reduces disease risk in a generalist plant pathogen invasion. LoGiudice et al. (2003) show that the preservation of vertebrate biodiversity and community composition can reduce the incidence of Lyme disease. Johnson et al. (2013) show that maintaining biodiversity can reduce disease transmission using integrate high-resolution field data with multi-scale experiments. Ostfeld et al. (2000) examine the function of biodiversity in the ecology of vector-borne zoonotic diseases. Particularly, they show that high species diversity can lead to a reduction in infection prevalence and disease risk in vertebrate communities under certain conditions. Keesing et al. (2010) argue based on current evidence that preserving intact ecosystems and their endemic biodiversity should generally reduce the prevalence of infectious diseases.

The dynamics of mixed resource ecosystems are complex and understanding the dynamics of such ecosystems is important for proper management and preservation. Mathematical models are one of the tools that can aid this process through exploring the possible dynamics of the systems. Nakazawa et al. (2012) developed a mathematical model analysis for plant disease dynamics co-mediated by herbivory. Their results from an eco-epidemeilogical perspective demonstarte that integration of tripartite interactions among host plant, plant pathogens and herbivour vectors is helpfull for sucssesful control of plant diseases. Here, we consider a more complex mathematical model to investigate the impact of resource species diversity on the spread of diseases in a mixed resource terrestrial ecosystem and illustrate the mechanisms whereby species diversity could lead to a reduction in diseases. Thus, the primary aim here is to explain theoretically how species diversity could reduce certain infectious diseases in mixed resource terrestrial ecosystems.

\section{Methods}

\section{Consumer-resource model}

In this study we explore variations in biodiversity in terms of plant species richness. We develop a simple model of plant resources with animal consumers. The model is similar to that in Rosenzweig and MacArthur (1963) but here we extend it by introducing infection together with multiple resources that a consumer (or group of similar consumers) feed on. We assume that each type of resource is made up of two classes: a susceptible group $S_{i}$ and an infected group $I_{i}$ such that $S_{i}+I_{i}=N_{i}$ (all mathematical symbols used in this paper are listed with their meanings in Appendix 3 at the end). Each susceptible group $S_{i}$ grow logistically and decrease as a result of infections or removal by the consumers (Kooi et al., 2011; Rosenzweig and MacArthur, 1963). The infected group increases when the susceptible $S_{i}$ become infected and decreases through death or feeding by the consumers (Nakazawa et al., 2012). Transmission of disease from the infected group (plants) $I_{i}$ to susceptible plants $S_{i}$ can be through indirect mode of transmission (Campbell, 1996). This is a situation when the pathogens causing the disease are carried independently by natural agencies like wind, water, animals, insects, mites, nematodes, birds etc. from the infected to suscetible plants. For instance, bacteria and fungi can be spread by water or wind. On the other hand, one of the most common vectors of viruses are insects. So for this study, infcetious diseases of an ecosystem that can be transmitted by any of these indirect mode of transmission are considered. Thus, it is assumed that the pathogen density, 
denoted by $P$, grow through the infected resources at rates $v_{i}$ and die at a rate $\xi$, and is how $S_{i}$ and $I_{i}$ interact. Resource consumption is modelled by hyperbolic response functions (Turchin, 2003). Based on these assumptions, we obtain the model (Eq. l)

$$
\begin{aligned}
& \frac{d N_{1}}{d t}=N_{1} r_{1}\left(1-\frac{N_{1}}{K_{1}}\right)-\frac{\alpha_{1} N_{1} Y}{\beta_{1}+N_{1}}-\gamma_{1} I_{1}, \\
& \frac{d S_{1}}{d t}=N_{1} r_{1}\left(1-\frac{N_{1}}{K_{1}}\right)-\frac{\alpha_{1} S_{1} Y}{\beta_{1}+N_{1}}-\sigma_{1} S_{1} P+\gamma_{1} I_{1}, \\
& \frac{d I_{1}}{d t}=\sigma_{1} S_{1} P-\frac{\alpha_{1} I_{1} Y}{\beta_{1}+N_{1}}-\left(\gamma_{1}+\mu_{1}\right) I_{1}, \\
& \cdot \\
& \frac{d N_{n}}{d t}=N_{n} r_{n}\left(1-\frac{N_{n}}{K_{n}}\right)-\frac{\alpha_{n} N_{n} Y}{\beta_{n}+N_{n}}-\gamma_{n} I_{n}, \\
& \frac{d S_{n}}{d t}=N_{n} r_{n}\left(1-\frac{N_{n}}{K_{n}}\right)-\frac{\alpha_{n} S_{n} Y}{\beta_{n}+N_{n}}-\sigma_{n} S_{n} P+\gamma_{n} I_{n}, \\
& \frac{d I_{n}}{d t}=\sigma_{n} S_{n} P-\frac{\alpha_{n} I_{n} Y}{\beta_{n}+N_{n}}-\left(\gamma_{n}+\mu_{n}\right) I_{n}, \\
& \frac{d P}{d t}=\sum_{i=1}^{n} v_{i} I_{i}-\xi P, \\
& \frac{d Y}{d t}=\sum_{i=1}^{n} \frac{c_{i} \alpha_{i} N_{i} Y}{\beta_{i}+N_{i}}-\tau Y,
\end{aligned}
$$

Note that $n$ is the number of resources and $\sum_{i=1}^{n} S_{i}=S, \sum_{i=1}^{n} I_{i}=I$ with the total density $N=S+I$. The meaning of variables and parameters are given in Table 1. We assume that infections are not transmitted from resources to consumer and that consumers interact with all resources uniformly. We do not consider equations for $\frac{d S_{i}}{d t}$ in our mathematical analyses, as $S_{i}$ can be easily determined from the already determined $N_{i}$ and $I_{i}$. Note, increasing resource diversity for this model system is achieved by increasing $n$. All variables are in the units of biomass $\left(\mathrm{g} / \mathrm{m}^{2}\right)$. Also, as the aim is to test the effects of resource diversity on the dynamics, the consumers are treated as one total biomass.

\section{Consumer-resource model for one resource}

To analyse the model, we start with the case where there is only one resource. For this homogenous case we drop the subscripts as there is only one resource (i.e. $\sum_{i=1}^{1} S_{i}=S, \sum_{i=1}^{1} I_{i}=I$ with the total density $N=S+I$ as before). We now obtain Equation 2: 


$$
\begin{aligned}
& \frac{d N}{d t}=N r\left(1-\frac{N}{K}\right)-\frac{\alpha N Y}{\beta+N}-\gamma I, \\
& \frac{d I}{d t}=\sigma S P-\frac{\alpha I Y}{\beta+N}-(\gamma+\mu) I, \\
& \frac{d P}{d t}=v I-\xi P, \\
& \frac{d Y}{d t}=\frac{c \alpha N Y}{\beta+N}-\tau Y,
\end{aligned}
$$

where variables and parameters have the same meanings as the corresponding ones defined in Table 1.

Table 1. Variables and parameters for model Equation 1

\begin{tabular}{c|c|c}
\hline Variables & Meaning & Units \\
\hline$S i$ & Density of susceptible resources $i$ & $\mathrm{~g} / \mathrm{m}^{2}$ \\
$N_{i}$ & Density of infected resources $i$ & $\mathrm{~g} / \mathrm{m}^{2}$ \\
$Y$ & Total density of resources $i, S_{i}+I_{i}$ & $\mathrm{~g} / \mathrm{m}^{2}$ \\
$P$ & Density of consumer & $\mathrm{g} / \mathrm{m}^{2}$ \\
\hline Parameters & Measure of foliar diseases pathogen & Units \\
\hline$r_{i}$ & Meaning & $/$ year \\
$K_{i}$ & Growth rate of resources $i$ & $\mathrm{~g} / \mathrm{m}^{2}$ \\
$\alpha_{i}$ & Carrying capacity of resources $i$ & $/$ year \\
$\beta_{i}$ & Ni removal by $Y i$ & $\mathrm{~g} / \mathrm{m}^{2}$ \\
$c$ & Ni when $\alpha i$ is half & Dimensionless \\
$\tau$ & Conversion of $S i$ or $I i$ into $Y$ & $/$ year \\
$\sigma_{i}$ & Reduction of $Y$ due to other factors & $/$ year \\
$\mu_{i}$ & Exposure of $S i$ to $P$ & $/$ year \\
$\gamma_{i}$ & Death rate of $I_{i}$ & $/$ year \\
$v_{i}$ & Recovery rate of $I_{i}$ & $/$ year \\
$\xi$ & Growth rate of $P$ (from infected $I i)$ & $/$ year \\
\hline
\end{tabular}

\section{Consumer-resource model with increased species diversity, $n=2$}

Next we increased the resource diversity by setting $n=2$ in model Equation 1 to obtain Equation 3: 


$$
\begin{aligned}
& \frac{d N_{1}}{d t}=N_{1} r_{1}\left(1-\frac{N_{1}}{K_{1}}\right)-\frac{\alpha_{1} N_{1} Y}{\beta_{1}+N_{1}}-\gamma_{1} I_{1}, \\
& \frac{d I_{1}}{d t}=\sigma_{1} S_{1} P-\frac{\alpha_{1} I_{1} Y}{\beta_{1}+N_{1}}-\left(\gamma_{1}+\mu_{1}\right) I_{1}, \\
& \frac{d N_{2}}{d t}=N_{2} r_{2}\left(1-\frac{N_{2}}{K_{2}}\right)-\frac{\alpha_{2} N_{2} Y}{\beta_{2}+N_{2}}-\gamma_{2} I_{2}, \\
& \frac{d I_{2}}{d t}=\sigma_{2} S_{2} P-\frac{\alpha_{2} I_{2} Y}{\beta_{2}+N_{2}}-\left(\gamma_{2}+\mu_{2}\right) I_{2}, \\
& \frac{d P}{d t}=\sum_{i=1}^{2} v_{i} I_{i}-\xi P, \\
& \frac{d Y}{d t}=\sum_{i=1}^{2} \frac{c_{i} \alpha_{i} N_{i} Y}{\beta_{i}+N_{i}}-\tau Y,
\end{aligned}
$$

a scenario where there are two distinct resources that the consumers can feed on.

\section{Threshold quantities used}

The basic reproduction number denoted by $R_{0}$ in epidemiology is a threshold quantity that gives a condition under which a disease can be eradicated or persist in the population (van den Driessche and Watmough, 2002; Tien and Earn, 2010). For $\mathrm{R}_{0}<1$ a disease can be eradicated but when $\mathrm{R}_{0}>1$ a disease persists. So, to ensure disease eradication mechanisms (natural or otherwise) are required that ensure the basic reproduction number is below unity. Epidemiologically, the basic reproduction number can also be used as a measure of the number of secondary infections in the system. For example, the basic reproduction number $R_{0}$ for Equation 2, using the next generation matrix method (van den Driessche and Watmough, 2002), is (Eq. 4):

$$
\mathrm{R}_{0}=\frac{\sigma v K}{\xi(\mu+\gamma)}
$$

The consumption number denoted by $C_{0}$ for a consumer-resource system model is a threshold quantity (similar to the basic reproduction number) that gives a condition under which the consumers will survive or not in the system (Collins and Duffy, 2016a). Here, when $\mathrm{C}_{0}<1$ consumers do not survive and when $\mathrm{C}_{0}>1$ consumers survive. So, coexistence of consumer and resources in the system requires that the consumption number is above unity. For example, the consumption number for Equation 2 can be calculated using the approach in Collins and Duffy (2016a) given by (Eq. 5):

$$
\mathrm{C}_{0}=\frac{c \alpha K}{\tau(\beta+K)}
$$

Mathematically, these two threshold quantities give conditions under which the equilibrium points of the system are stable and enable a qualitative analysis of the models. 


\section{Numerical simulations}

Numerical simulations are used to support our analytical predictions. In particular, the impact of resource diversity on the dynamics of infected resource biomass is considered. The parameter values used for the numerical simulations are given in Table 2.

Table 2. Parameter values used for model simulations with their source

\begin{tabular}{c|c|c|c}
\hline Variables/parameters & Value & Unit & Source \\
\hline$r$ & 0.014 & Duffy (2001) \\
\hline$K$ & 500 & $\mathrm{~g} / \mathrm{m}^{2}$ & Owen-Smith (2004); Duffy (2001) \\
\hline$\alpha$ & 0.025 & Duffy (2001) & Duffy (2001) \\
\hline$\beta$ & 40 & $\mathrm{~g} / \mathrm{m}^{2}$ & Estimate \\
\hline$\tau$ & 0.75 & Dimensionless & Owen-Smith (2004); Duffy (2001) \\
\hline$\gamma$ & 0.0175 & Estimate \\
\hline$\mu$ & 0.01 & /day & Estimate \\
\hline$v$ & 0.8 & /year & Estimate \\
\hline$\xi$ & 0.002 & /year & Estimate \\
\hline
\end{tabular}

We are interested in comparing the results of the models with increasing diversity and so to ensure these models are comparable we equal their biomasses by making:

$$
r_{1}=r_{2}=r, \alpha_{1}=\alpha_{2}=\alpha, K_{1}=K_{2}=K / 2, \mu_{1}=\mu_{2}=\mu, \gamma_{1}=\gamma_{2}=\gamma, \sigma_{1}=\sigma_{2}=\sigma \text {. }
$$

Based on these assumptions, the basic reproduction number of Equation 1 for $n=1$, $n=2$ and $n=3$ becomes equal. So to determine the impact of resource diversity on the spread of disease, we compare the dynamics of the models numerically under these assumptions.

These models are used to investigate the effect of increasing resource diversity. In most epidemiological modelling studies a fundamental result is that when the basic reproduction number is less than unity the infected populations dies out and when the basic reproduction number is greater than unity the disease persists (Castillo-Chavez et al., 2002; van den Driessche and Watmough, 2002; Tien and Earn, 2010). Ecologically, when the consumption number is less than unity, consumers will not survive and when the consumption number is greater than unity, consumers survive (Collins and Duffy, 2016a). So, the basic reproduction number and consumption number are bifurcation quantities. Thus, to investigate these scenarios numerically we consider parameter values such that the basic reproduction number and consumption number are less than and/or greater than unity. These various values of basic reproduction number and 
consumption number are obtained by varying the most sensitive parameter while the others are kept fixed. We vary $\tau$ to obtain various value of $C_{0}$ since it is the most sensitive parameter for $C_{0}$ and we vary $\sigma$ to obtain various value of $R_{0}$ since it is the most sensitive parameter for $R_{0}$.

\section{Analyses and results}

\section{Qualitative analyses}

The system with $n=1$ (Eq. 2) has many equilibrium points but we present only the ones crucial for our analyses, given by:

$$
\begin{aligned}
& \mathrm{E}_{1}=\left(N^{*}, I^{*}, P^{*}, Y^{*}\right)=(0,0,0,0), \\
& \mathrm{E}_{2}=\left(N^{*}, I^{*}, P^{*}, Y^{*}\right)=(\mathrm{K}, 0,0,0), \\
& \mathrm{E}_{3}=\left(N^{*}, I^{*}, P^{*}, Y^{*}\right)=\left(\frac{\tau \beta}{c \alpha-\tau}, 0, \frac{\mathrm{r}}{\alpha}\left(1-\frac{\mathrm{N}^{*}}{\mathrm{~K}}\right)\left(\beta+\mathrm{N}^{*}\right), 0\right), \quad \text { if } C_{0} \geq 1 .
\end{aligned}
$$

Note that $\mathrm{E}_{3}=\mathrm{E}_{2}$ if $\mathrm{C}_{0}=1$. This suggests that $C_{0}$ and $R_{0}$ are bifurcation quantities. The short- and long-term dynamics of Equation 2 can be described by the stability about the equilibrium points Liao and Wang (2011). Thus, the stability results of the model are summarized in the following theorem.

\section{Theorem 1}

(i) The trivial equilibrium point $\mathrm{E} 1$ is unstable.

(ii) The equilibrium point $\mathrm{E} 2$ is stable if $\mathrm{C} 0<1$ and $\mathrm{R} 0<1$.

(iii) For $\mathrm{C} 0>1$, the equilibrium point $\mathrm{E} 3$ is stable irrespective the value of $\mathrm{R} 0$.

The proof of Theorem 1 is given in Appendix 1.

Ecologically, based on this formulation of the system dynamics, Theorem 1 implies the following for $n=1$ : (i) resources, consumers and pathogens cannot all go extinct (ii) consumers, infected resources and pathogens can be eradicated when $C_{0}<1$ and $R_{0}<1$. (iii) for $C_{0}>1$, infected resources can be eradicated irrespective the value of $R_{0}$ (i.e. for $R_{0}<1$ or $R_{0}>1$ ). Ecologically, co-existence of resources and consumers can occur in a system only when $C_{0}>1$. Thus, the consumption number $C_{0}$ and the basic reproduction number $R_{0}$ are important bifurcation quantities in the dynamical system analyses of Equation 2.

We present a qualitative analyse of the model for $n=2(E q .3)$. Again, the system has many equilibrium points, but we analyse further the crucial ones representing disease eradication:

$$
\begin{aligned}
& \mathrm{E}_{1}=\left(N_{1}{ }^{*}, I_{1}{ }^{*}, N_{2}{ }^{*}, I_{2}{ }^{*}, P^{*}, Y^{*}\right)=(0,0,0,0,0,0), \\
& \mathrm{E}_{2}=\left(N_{1}{ }^{*}, I_{1}{ }^{*}, N_{2}{ }^{*}, I_{2}{ }^{*}, P^{*}, Y^{*}\right)=\left(\mathrm{K}_{1}, 0, \mathrm{~K}_{2}, 0,0,0\right), \\
& \mathrm{E}_{3}=\left(N_{1}{ }^{*}, I_{1}{ }^{*}, N_{2}{ }^{*}, I_{2}{ }^{*}, P^{*}, Y^{*}\right)=\left(\mathrm{N}^{*}{ }_{1}, 0, \mathrm{~N}^{*}{ }_{2}, 0,0, \mathrm{Y}^{*}\right),
\end{aligned}
$$


where $\mathrm{N}_{1}^{*} \quad$ and $\mathrm{N}_{2}^{*} \quad$ are such that $\frac{\alpha_{1} N_{1}^{*}}{\beta_{1}+N_{1}^{*}}+\frac{\alpha_{2} N_{2}^{*}}{\beta_{2}+N_{2}^{*}}=\frac{\tau}{\mathrm{c}} \quad$ and $Y^{*}=\frac{r_{1}}{\alpha_{1}}\left(1-\frac{N_{1}^{*}}{K_{1}}\right)\left(\beta_{1}+N_{1}^{*}\right)$ or $Y^{*}=\frac{r_{2}}{\alpha_{2}}\left(1-\frac{N_{2}^{*}}{K_{2}}\right)\left(\beta_{2}+N_{2}^{*}\right)$.

The basic reproduction number for $n=2$ is calculated, again using the next generation matrix method (see Appendix 2) as (Eq. 6)

$$
\bar{R}_{0}=\frac{R_{11}+R_{22}+\sqrt{\left(R_{11}+R_{22}\right)^{2}+4\left(R_{12} R_{21}-R_{11} R_{22}\right)}}{2},
$$

where $R_{11}=\frac{\sigma_{1} v_{1} K_{1}}{\xi\left(\mu_{1}+\gamma_{1}\right)}, R_{12}=\frac{\sigma_{1} v_{2} K_{1}}{\xi\left(\mu_{2}+\gamma_{2}\right)}, R_{21}=\frac{\sigma_{2} v_{1} K_{2}}{\xi\left(\mu_{2}+\gamma_{2}\right)}, R_{22}=\frac{\sigma_{2} v_{2} K_{2}}{\xi\left(\mu_{2}+\gamma_{2}\right)}$.

Since $R_{12} R_{21}=R_{11} R_{22}$, the basic reproduction number $R_{0}$ can be simplified as (Eq. 7)

$$
\bar{R}_{0}=R_{11}+R_{22}
$$

Mathematically, the quantities $R_{11}$ and $R_{22}$ can be regarded as the basic reproduction numbers associated with resources 1 and 2 respectively.

The consumption number (threshold quantity) denoted by $\bar{C}_{0}$ for $n=2$ can be calculated using the approach in Collins and Duffy (2016a) and is given by (Eq. 8)

$$
\bar{C}_{0}=C_{1}+C_{2},
$$

where $C_{1}=\frac{c \alpha_{1} K_{1}}{\tau\left(\beta_{1}+K_{1}\right)}$ and $C_{2}=\frac{c \alpha_{2} K_{2}}{\tau\left(\beta_{2}+K_{2}\right)}$ are consumption numbers associated with resources 1 and 2 respectively.

Now again the dynamical system analyses are carried out utilizing the concept of the basic reproduction number $R_{0}$ together with the consumption number $\bar{C}_{0}$ where necessary. The dynamics of Equation 3 can be summarized in the following stability results given in the theorem below.

Theorem 2

(i) The trivial equilibrium points $\mathrm{E} 1$ is unstable.

(ii) The equilibrium point $\mathrm{E}_{2}$ is stable if $\bar{C}_{0}<1$ and $\bar{R}_{0}<1$.

(iii) For $\bar{C}_{0}>1$, the equilibrium point $\mathrm{E}_{3}$ is stable irrespective the value of $\bar{R}_{0}$.

The proof of Theorem 2 (i) and (ii) can be established using the approach used in the proof of Theorem 1. For (iii), the proof of the special case of equivalent parameter values (see above) is also easily established. For the general case, the ensuing equations 
are too complicated to solve. However, all numerical simulations performed by us support this proposition in general.

Comparing the consumption numbers of the models for $n=1$ and $n=2$, using the assumptions of equal biomasses, the consumption number for $n=2$ is always greater than the consumption number for $n=1$. This can be illustrated mathematically as follows:

$$
\begin{aligned}
\bar{C}_{0} & =C_{1}+C_{2} \\
& =\frac{c \alpha_{1} K_{1}}{\tau\left(\beta_{1}+K_{1}\right)}+\frac{c \alpha_{2} K_{2}}{\tau\left(\beta_{2}+K_{2}\right)} \\
& =\frac{c \alpha K}{\tau(\beta+K / 2)} \\
& >\frac{c \alpha K}{\tau(\beta+K)}=C_{0}
\end{aligned}
$$

Thus (Eq.9),

$$
\bar{C}_{0}>C_{0}
$$

Similarly, we can show that this result also holds for any number of species $n$. So, larger values of $n$ result in larger values of the consumption number. Thus, an increase in resource diversity $n$ will lead to an increase in the consumption number. It can be shown that for $n=1$ increasing the consumption number can result in a decrease in the infected biomass. Here increasing diversity is shown to result in an increase in the value of the consumption number. Thus, based on these results, increasing resource diversity as given here should lead to a decrease in the infected biomass in general. This possibility is explored numerically in the next section.

\section{Numerical simulation results}

The results of our numerical simulations are given in Figure 1, using the parameter values in Table 2. For Figure 1 we set $\tau=0.032$ which correspond to $C_{0}=0.5425<1$ and different basic reproduction numbers are calculated by varying $\sigma\left(R_{0}=0.5143\right.$, $0.9772,2.5717,5.1434$ using $\sigma=0.001,0.0019,0.005,0.01$ respectively).

From Figure $1 a$ and $b$ the biomass of the infected resources decrease when $n=1$ and $n=2$, for $R_{0}<1$. For the case when $n=2$, the infected biomass decreases faster. In Figure $1 c$ and $d$ when $R_{0}>1$, the biomass of infected resources increases for both $n=1$ and $n=2$, but increases faster for $n=1$. When $n=3$ these results change fundamentally (Fig. $1 c$ and $d$ ) in that even for $R_{0}>1$ the infected biomass is eradicated (note that the oscillation in the figure eventually dampen to zero). Increasing the consumption number to $C_{0}>1$ by varying $\tau$ strengthens this result in that now for $n=2$ the infected biomass is eradicated as well (not shown).

\section{Analyses of the general consumer-resource model, Equation 1}

In this section, we show how some of the results of the previous sections can be extended to the general case. 
The consumption number (threshold quantity) denoted by $C_{0}$ for the general model Equation 1 can be calculated using the approach in Collins and Duffy (2016a) and is given by $(E q .10)$

$$
\bar{C}_{0}=\sum_{i=1}^{n} C_{i}
$$

where $C_{i}=\frac{c \alpha_{i} K_{i}}{\tau\left(\beta_{i}+K_{i}\right)}$ is the consumption number associated with resource $i$.

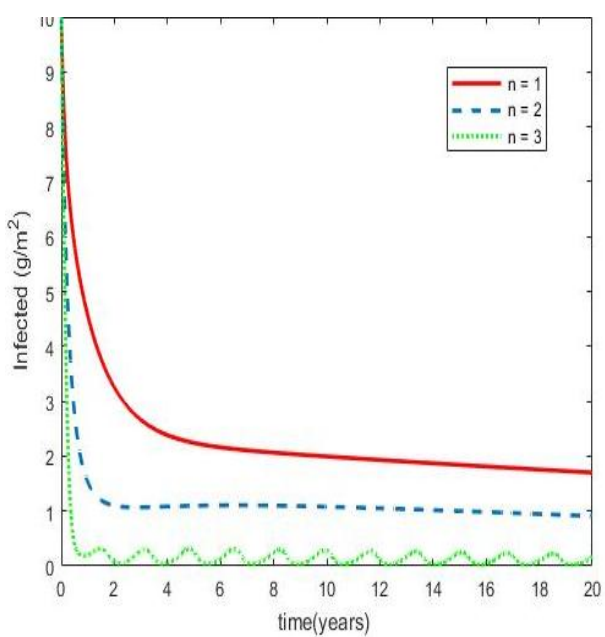

a

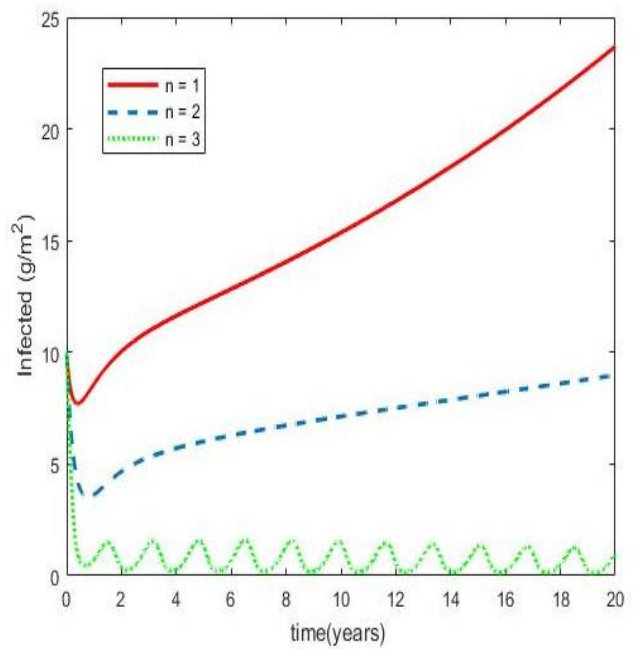

c

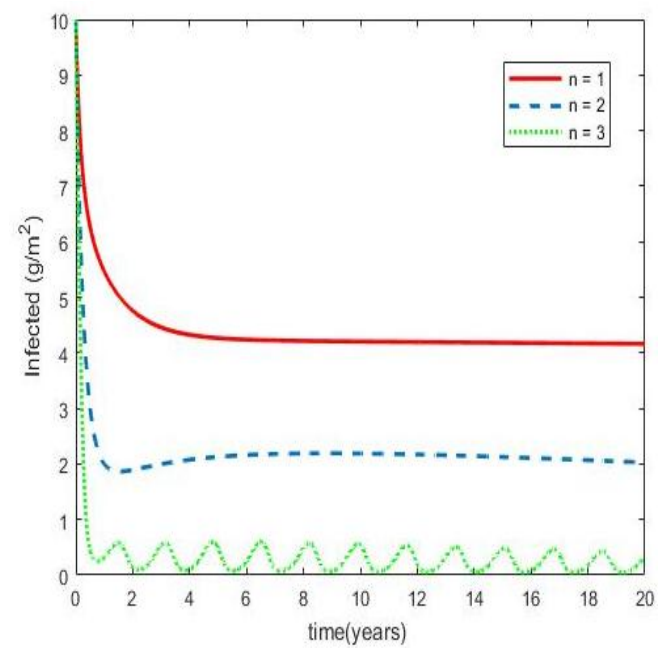

b

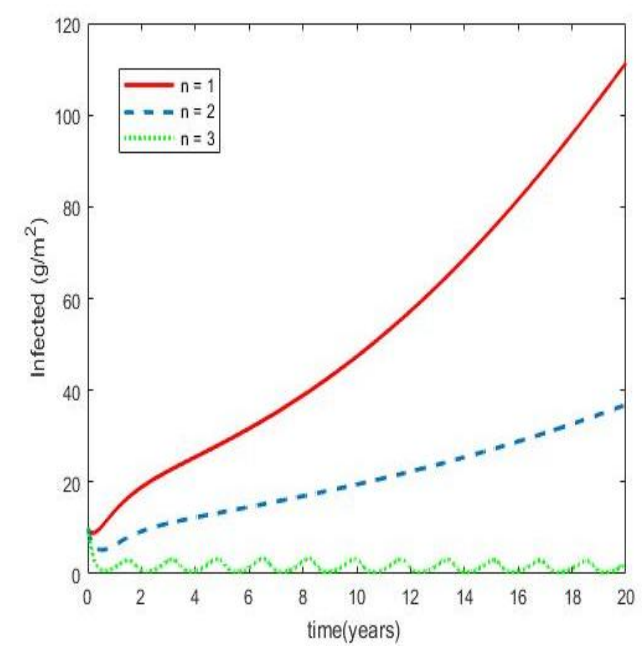

d

Figure 1. Plot illustrating the possible difference on the dynamics of model Equation 1 for $n=1, n=2$ and $n=3$ for $C_{0}=0.5425<1$ and for various values of $R_{0}:(a) R_{0}=0.5143(b)$ $R_{0}=0.9772(c) R_{0}=2.5717(d) R_{0}=5.1434$ (see text for details)

The basic reproduction number of the more general model Equation 1 can also be determined using the next generation matrix (see Appendix 2). The basic reproduction number $R_{0}$ for this general case is difficult to calculate analytically. However, if we 
make the same assumptions used in the numerical simulations but for any $n$, the basic reproduction number $R_{0}$ becomes (Eq. 11)

$$
\bar{R}_{0}=\sum_{i=1}^{n} R_{i i}
$$

It is easy to see that for this general model, the basic reproduction number is also uniform irrespective of $n$ (magnitude of resource diversity) under the same assumptions for equal biomass as in the cases of $n=1, n=2$ and $n=3$. By similar reasoning, the larger the value of $n$, the larger the consumption number.

\section{Discussion}

A growing understanding of the benefits of biodiversity conservation is important for improved policies toward enhancing human well-being. In this study, we explored one of a potential benefit of ecosystem biodiversity where there are infectious diseases. By formulating and analysing an appropriate mathematical consumer-resource model that takes resource diversity into consideration, we were able to show how resource diversity could limit the spread of infectious diseases. Our results are based on qualitative analyses supported by numerical simulations.

A general consumer-resource model with $n$ multiple types of resources representing resource diversity with these resources exposed to infectious disease was developed. Our analyses consider cases with $n=1$ and $n>1$ (i.e. a situation where there is only one type of resource and situations with more resources). The important mathematical features of the models such as the basic reproduction number and consumption number were obtained and used to investigate the dynamics. The results of the dynamical system analyses of these models using these concepts agree in general with results in epidemiology and consumer-resource system (Castillo-Chavez et al., 2002; van den Driessche and Watmough, 2002; Tien and Earn, 2010; Collins and Duffy, 2016a, b). In particular, we use these threshold quantities to consider the effects of diversity on the spread of disease. In all the cases considered increasing resource diversity reduced infected resources.

Our numerical results for $n=1$ and $n=2$, where $R_{0}<1$ agree with a fundamental result in epidemiological modelling that when the basic reproduction number is less than one infected populations die out (Castillo-Chavez et al., 2002; van den Driessche and Watmough, 2002; Tien and Earn, 2010). However, for the case when $n=2$ we shown that the infected biomass decreases faster. Where $R_{0}>1$, the biomass of infected resources increase as expected. However, we show that it increased faster for $n=1$ compared to $\mathrm{n}=2$. When $n=3$ these results change fundamentally in that even for $R_{0}>1$ the infected biomass is eradicated. Increasing the consumption number to $C_{0}>1$ by varying $\tau$ strengthens this result in that then even for $n=2$ the infected biomass can be eradicated. Overall, these results support our analytical predictions that increasing resource diversity leads to a decrease in the biomass of infected resources. However, the most interesting result is that, for a case when infected plants are expected to persist, increased diversity results in infected resources dying out. To reiterate, we show cases where even for $R_{0}>1$ then infected resources are eliminated for $C_{0}>1$ large enough.

For the general model (Eq. I), conditions are shown, under the assumption of equal biomass, where an increase in resource diversity $n$ will lead to an increase in the 
consumption number. It is expected that the dynamics of this general model will also be governed by its corresponding basic reproduction number and consumption number (Heesterbeek and Roberts, 2007; Roberts and Heesterbeek, 2003; Collins and Duffy, 2016b). Consequently, our earlier findings that increasing diversity could lead to a decrease in the spread of infectious disease should also hold for this general model.

The models suggested here have many simplifying assumptions. However, as a first step they represent a method to compare ecosystems with different levels of diversity (here plant species richness). It is shown how increasing diversity in this system can help in reducing infectious disease in the system. Thus, increasing resource diversity through biodiversity conservation could promote healthier systems. This possibility could be important for both the environmental and agricultural future of the planet.

\section{Conclusions}

Biodiversity conservation has been shown to reduce the spread of some diseases in various ways (Haas et al., 2011; LoGiudice et al., 2003; Johnson et al., 2013; Ostfeld et al., 2000; Keesing et al., 2010). This study aimed to explain theoretically how biodiversity, through resource diversity, could lead to reduction in the spread of infectious diseases in a mixed resource ecosystem and the results are in the main consistent with this literature. As such, models of this kind with more and more detailed information can be considered to explain other aspects of system dynamics. Our model illustration here that resource diversity can lead to disease eradication is, as far as we know, new. Thus, this study complements results in this area and at the same time points to further research.

Acknowledgements. This work is based on research supported in part by the National Research Foundation of South Africa (Grant Numbers 98892 and 85494).

\section{REFERENCES}

[1] Auger, P., Mchich, R., Chowdhury, T., Sallet, G., Tchuente, M., Chattopadhyay, J. (2009): Effects of a disease affecting a predator on the dynamics of a predator-prey system. - Journal of Theoretical Biology 258(3): 344-351.

[2] Bai, Y., Han, X., Wu, J., Chen, Z., Li, L. (2004): Ecosystem stability and compensatory effects in the Inner Mongolia grassland. - Nature 431: 181-184.

[3] Campbell, R. N. (1996): Fungal transmission of plant viruses. - Annual Review of Phytopathology 34(1): $87-108$.

[4] Castillo-Chavez, C., Feng, Z., Huang, W. (2002): On the Computation of $\mathrm{R}_{0}$ and Its Role on Global Stability. - In: Bies, D., Blower, S., Driessche, P. van d., Kirschner, D., Yakubu, A.-A. (eds.) Mathematical Approaches for Emerging and Re-emerging Infectious Diseases: An Introduction. IMA Vol. 125. Springer-Verlag, New York.

[5] Collins, O. C., Duffy, K. J. (2016a): Consumption threshold used to investigate stability and ecological dominance in consumer-resource dynamics. - Ecological Modelling 319: $155-162$.

[6] Collins, O. C., Duffy, K. J. (2016b): Understanding multiple species ecosystem dynamics using a consumer resource model. - Natural Resource Modeling 29(1): 145-158.

[7] Duffy, K. J. (2011): Simulations to investigate animal movement effects on population dynamics. - Natural Resource Modeling 24(1): 48-60. 
[8] Ezenwa, V. O., Godsey, M. S., King, R. J., Guptill, S. C. (2006): Avian diversity and West Nile virus: testing associations between biodiversity and infectious disease risk. Proceedings of the Royal Society of London B: Biological Sciences 273(1582): 109-117.

[9] Haas, S. E., Hooten, M. B., Rizzo, D. M., Meentemeyer, R. K. (2011): Forest species diversity reduces disease risk in a generalist plant pathogen invasion. - Ecology Letters 14(11): 1108-1116.

[10] Hautier, Y., Tilman, D., Isbell, F., Seabloom, E. W., Borer, E. T., Reich, P. B. (2015): Anthropogenic environmental changes affect ecosystem stability via biodiversity. Science 348(6232): 336-340.

[11] Heesterbeek, J. A. P., Roberts, M. G. (2007): The type-reproduction number T in models for infectious disease control. - Mathematical Biosciences 206(1): 3-10.

[12] Isbell, F., Craven, D., Connolly, J., Loreau, M., Schmid, B., Beierkuhnlein, C., Bezemer, T. M., Bonin, C., Bruelheide, H., De Luca, E., Ebeling, A. (2015): Biodiversity increases the resistance of ecosystem productivity to climate extremes. - Nature 526(7574): 574577.

[13] Johnson, P. T., Preston, D. L., Hoverman, J. T., Richgels, K. L. (2013): Biodiversity decreases disease through predictable changes in host community competence. - Nature 494(7436): 230-233.

[14] Keesing, F., Belden, L. K., Daszak, P., Dobson, A., Harvell, C. D., Holt, R. D., Hudson, P., Jolles, A., Jones, K. E., Mitchell, C. E., Myers, S. S. (2010): Impacts of biodiversity on the emergence and transmission of infectious diseases. - Nature 468(7324): 647-652.

[15] Kooi, B. W., van Voorn, G. A., pada Das, K. (2011): Stabilization and complex dynamics in a predator-prey model with predator suffering from an infectious disease. - Ecological Complexity 8(1): 113-122.

[16] Liao, S., Wang, J. (2011): Stability analysis and application of a mathematical cholera model. - Mathematical Biosciences and Engineering 8(3): 733-752.

[17] LoGiudice, K., Ostfeld, R. S., Schmidt, K. A., Keesing, F. (2003): The ecology of infectious disease: effects of host diversity and community composition on Lyme disease risk. - Proceedings of the National Academy of Sciences 100(2): 567-571.

[18] Naeem, S. (ed.) (2009): Biodiversity, Ecosystem Functioning, and Human Wellbeing: An Ecological and Economic Perspective. - Oxford University Press, Oxford.

[19] Naeem, S., Li, S. (1997): Biodiversity enhances ecosystem reliability. - Nature 390(6659): 507-509.

[20] Nakazawa, T., Yamanaka, T., Urano, S. (2012): Model analysis for plant disease dynamics co-mediated by herbivory and herbivore-borne phytopathogens. - Biology Letters 8(4): 685-688.

[21] Nunes, P. A. L. D., Ding, H., Boteler, B., ten Brink, P., Cottee-Jones, E., Davis, M., Ghermandi, A., Kaphengst, T., Lago, M., McConville, A. J., Naumann, S., Pieterse, M., Rayment, M., Varma, A. (2011): The Social Dimension of Biodiversity Policy: Final Report for the European Commission, DG Environment under Contract: ENV.G.1/FRA/2006/0073 2nd, pp. vii-205. - Venice/Brussels, February 2011.

[22] Ostfeld, R. S., Keesing, F. (2000): Biodiversity series: the function of biodiversity in the ecology of vector-borne zoonotic diseases. - Canadian Journal of Zoology 78(12): 20612078.

[23] Ostfeld, R. S., Keesing, F. (2012): Effects of host diversity on infectious disease. Annual Review of Ecology, Evolution, and Systematics 43: 157-182.

[24] Owen-Smith, N. (2004): Functional heterogeneity in resources within landscapes and herbivore population dynamics. - Landscape Ecology 19(7): 761-771.

[25] Pada Das, K., Kundu, K., Chattopadhyay, J. (2011): A predator-prey mathematical model with both the populations affected by diseases. - Ecological Complexity 8(1): 68-80.

[26] Patz, J. A., Confalonieri, U. E. C., Amerasinghe, F. P., Chua, K. B., Daszak, P., Hyatt, A. D., Molyneux, D., Thomson, M., Yameogo, L., Lazaro, M. M., Vasconcelos, P., RubioPalis, Y., Campbell-Lendrum, D., Jaenisch, T., Mahamat, H., Mutero, C., Waltner- 
Toews, D., Whiteman, C. (2005): Human Health: Ecosystem Regulation of Infectious Diseases. - In: Hassan, R., Scholes, R., Ash, N. (eds.) Ecosystems and Human WellBeing. Current State and Trends, Vol. 1. The Millennium Ecosystem Assessment, Chap. 14, pp. 391-415. Island Press, Washington, DC.

[27] Pimentel, D., Wilson, C., McCullum, C., Huang, R., Dwen, P., Flack, J., Tran, Q., Saltman, T., Cliff, B. (1997): Economic and environmental benefits of biodiversity. BioScience 47(11): 747-757.

[28] Roberts, M. G., Heesterbeek, J. A. P. (2003): A new method for estimating the effort required to control an infectious disease. - Proceedings of the Royal Society of London B: Biological Sciences 270(1522): 1359-1364.

[29] Rosenzweig, M. L., MacArthur, R. H. (1963): Graphical representation and stability conditions of predator-prey interactions. - The American Naturalist 97(895): 209-223.

[30] Tewa, J. J., Djeumen, V. Y., Bowong, S. (2013): Predator-prey model with Holling response function of type II and SIS infectious disease. - Applied Mathematical Modelling 37(7): 4825-4841.

[31] Tien, J. H., Earn, D. J. (2010): Multiple transmission pathways and disease dynamics in a waterborne pathogen model. - Bulletin of Mathematical Biology 72(6): 1506-1533.

[32] Turchin, P. (2003): Complex Population Dynamics: A Theoretical/Empirical Synthesis (Vol. 35). - Princeton University Press, Princeton, NJ.

[33] Van den Driessche, P., Watmough, J. (2002): Reproduction numbers and sub-threshold endemic equilibria for compartmental models of disease transmission. - Mathematical Biosciences 180(1): 29-48.

[34] World Health Organization (WHO) (2016): Zoonoses and the Human-AnimalEcosystems Interface. - http://www.who.int/zoonoses/en/ (May 2016). 


\section{Appendix 1}

\section{Proof of Theorem 1}

The proof of Theorem 1 is given as follows:

(i) The Jacobian of Equation 2 evaluated at the trivial equilibrium point E1 has the following eigenvalues:

$$
\lambda_{1}=\mathrm{r}, \lambda_{2}=-(\mu+\gamma), \lambda_{3}=-\xi, \lambda_{4}=-\tau \text {. }
$$

Thus, $\mathrm{E}_{1}$ is unstable since $\lambda_{1}=r>0$.

(ii) The Jacobian of Equation 2 evaluated at the equilibrium point E2 has the following eigenvalues:

$$
\lambda_{1}=-\mathrm{r}, \lambda_{2}=\tau\left(C_{0}-1\right), \lambda_{3,4}=\frac{-(\gamma+\mu+\xi) \pm \sqrt{(\gamma+\mu+\xi)^{2}+4 \xi(\gamma+\mu)\left(R_{0}-1\right)}}{2} \text {. }
$$

Clearly, $\lambda_{2}<\Leftrightarrow \mathrm{C}_{0}<1$ and $\lambda_{3,4}<\Leftrightarrow \mathrm{R}_{0}<1$. Thus the equilibrium point $\mathrm{E}_{1}$ is stable if $\mathrm{C}_{0}<1$ and $\mathrm{R}_{0}<1$.

(iii) The Jacobian of model Equation 2 evaluated at the equilibrium point $\mathrm{E}_{3}$ has the following eigenvalues:

$$
\lambda_{1,2}=\frac{-\left(a_{22}+\xi\right) \pm \sqrt{\left(a_{22}+\xi\right)^{2}-4\left(a_{22} \xi-a_{23} v\right)}}{2}, \lambda_{3,4}=\frac{-a_{11} \pm \sqrt{a_{11}{ }^{2}-4 a_{14} a_{41}}}{2},
$$

where

$$
a_{11}=\frac{r N^{*}}{K\left(\beta+N^{*}\right)}\left(\beta+2 N^{*}-K\right), a_{22}=\gamma+\mu+\frac{\alpha Y^{*}}{\beta+N^{*}}, \quad a_{23}=\sigma N^{*},
$$

$a_{14}=\frac{\alpha N^{*}}{\beta+N^{*}}$ and $a_{41}=\frac{\alpha c \beta N^{*}}{\left(\beta+N^{*}\right)^{2}}$. We observe that $\lambda_{12}<0 \Leftrightarrow a_{22} \xi-a_{23} v>0$. By elementary algebraic calculations we have that $a_{22} \xi-a_{23} v>0$. Next, $\lambda_{3,4}<0 \Leftrightarrow a_{11}<0$. Similarly, we can show by elementary algebraic calculations that $a_{11}<0 \Leftrightarrow C_{0}>0$. Thus, for $C_{0}>1$, the equilibrium point $\mathrm{E}_{3}$ is stable irrespective the value of $R_{0}$. 


\section{Appendix 2}

\section{Calculations of the basic reproduction number}

For $n=2$

The basic reproduction number for $n=2$ is calculated, again using the next generation matrix approach of van den Driessche and Watmough (2002). The associated next generation matrices are given by

$$
F=\left(\begin{array}{ccc}
0 & 0 & K_{1} \sigma_{1} \\
0 & 0 & K_{2} \sigma_{2} \\
0 & 0 & 0
\end{array}\right), V=\left(\begin{array}{ccc}
\mu_{1}+\gamma_{1} & 0 & 0 \\
0 & \mu_{2}+\gamma_{2} & 0 \\
-v_{1} & -v_{2} & \xi
\end{array}\right)
$$

with

$$
F V^{-1}=\left(\begin{array}{ccc}
R_{11} & R_{12} & \frac{K_{1} \sigma_{1}}{\xi} \\
R_{21} & R_{22} & \frac{K_{2} \sigma_{2}}{\xi} \\
0 & 0 & 0
\end{array}\right),
$$

where $R_{11}=\frac{\sigma_{1} v_{1} K_{1}}{\xi\left(\mu_{1}+\gamma_{1}\right)}, R_{12}=\frac{\sigma_{1} v_{2} K_{1}}{\xi\left(\mu_{2}+\gamma_{2}\right)}, R_{21}=\frac{\sigma_{2} v_{1} K_{2}}{\xi\left(\mu_{2}+\gamma_{2}\right)}, R_{22}=\frac{\sigma_{2} v_{2} K_{2}}{\xi\left(\mu_{2}+\gamma_{2}\right)}$.

The basic reproduction number $R_{0}$ for model Equation 3 is now given by the dominant eigenvalue of the next generation matrix $F V^{-1}$ and is

$$
\bar{R}_{0}=\frac{R_{11}+R_{22}+\sqrt{\left(R_{11}+R_{22}\right)^{2}+4\left(R_{12} R_{21}-R_{11} R_{22}\right)}}{2} .
$$

For the general model, Equation 1

The basic reproduction number of the more general model Equation 1 can also determined using the next generation matrix approach of van den Driessche and Watmough (2002). The associated next generation matrices are given by

$$
F=\left(\begin{array}{ccccc}
0 & 0 & \ldots & 0 & K_{1} \sigma_{1} \\
0 & 0 & \ldots & 0 & K_{2} \sigma_{2} \\
\cdot & & & & \\
. & & & & \\
0 & 0 & \ldots & 0 & K_{n} \sigma_{n} \\
0 & 0 & \ldots & 0 & 0
\end{array}\right), V=\left(\begin{array}{ccccccc}
\gamma_{1}+\mu_{1} & 0 & 0 & 0 & \ldots & 0 \\
0 & \gamma_{2}+\mu_{2} & 0 & 0 & \ldots & 0 \\
. & & & & & \\
. & & & & & \\
0 & 0 & \ldots & 0 & \gamma_{n}+\mu_{n} & 0 \\
-v_{1} & -v_{2} & -v_{3} & \ldots & -v_{n} & \xi
\end{array}\right) \text {, }
$$


with $F V^{-1}=\left(\begin{array}{ccccc}R_{11} & R_{12} & \ldots & R_{1 n} & \frac{K_{1} \sigma_{1}}{\xi} \\ R_{21} & R_{22} & \ldots & R_{2 n} & \frac{K_{2} \sigma_{2}}{\xi} \\ \cdot & & & & \\ \cdot & & & & \\ R_{n 1} & R_{n 2} & \ldots & R_{n n} & \frac{K_{n} \sigma_{n}}{\xi} \\ 0 & 0 & \ldots & 0 & 0\end{array}\right)$,

where $R_{i j}=\frac{\sigma_{i} v_{j} K_{i}}{\xi\left(\mu_{j}+\gamma_{j}\right)}$. The basic reproduction number $R_{0}$ is as in the previous cases $n=2$ or $n=1$ is the dominant eigenvalue of $F V^{-1}$. For this general case, the dominant eigenvalues are difficult to calculate analytically. However, if we take the same assumptions used in the numerical simulation but for any $n$, the basic reproduction number $R_{0}$ becomes

$$
\bar{R}_{0}=\sum_{i=1}^{n} R_{i i}
$$




\section{Appendix 3}

\section{List of all symbols used}

\begin{tabular}{|c|c|c|}
\hline Variables & Meaning & Units \\
\hline$S i$ & Density of susceptible resources $i$ & $\mathrm{~g} / \mathrm{m}^{2}$ \\
\hline$S$ & Total density of susceptible resource & $\mathrm{g} / \mathrm{m}^{2}$ \\
\hline Ii & Density of infected resources $i$ & $\mathrm{~g} / \mathrm{m}^{2}$ \\
\hline$I$ & Total density of infected resource & $\mathrm{g} / \mathrm{m}^{2}$ \\
\hline$N_{i}$ & Total density of resources $i, S_{i}+I_{i}$ & $\mathrm{~g} / \mathrm{m}^{2}$ \\
\hline$N$ & Total density resource, $S+I$ & $\mathrm{~g} / \mathrm{m}^{2}$ \\
\hline$Y$ & Density of consumer & $\mathrm{g} / \mathrm{m}^{2}$ \\
\hline$P$ & Measure of foliar diseases pathogen & $\mathrm{g} / \mathrm{m}^{2}$ \\
\hline Parameters & Meaning & Units \\
\hline$r_{i}$ & Growth rate of resources $i$ & /year \\
\hline$K_{i}$ & Carrying capacity of resources $i$ & $\mathrm{~g} / \mathrm{m}^{2}$ \\
\hline$\alpha_{i}$ & Ni removal by $Y i$ & /year \\
\hline$\beta_{i}$ & Ni when $\alpha i$ is half & $\mathrm{g} / \mathrm{m}^{2}$ \\
\hline$c$ & Conversion of $S i$ or $I i$ into $Y$ & Dimensionless \\
\hline$\tau$ & Reduction of $Y$ due to other factors & /year \\
\hline$\sigma_{i}$ & Exposure of $S i$ to $P$ & /year \\
\hline$\mu_{i}$ & Death rate of $I_{i}$ & /year \\
\hline$\gamma_{i}$ & Recovery rate of $I_{i}$ & /year \\
\hline$v_{i}$ & Growth rate of $P$ (from infected $I i$ ) & /year \\
\hline$\xi$ & Death rate of $P$ & /year \\
\hline Other mathematical symbols & \multicolumn{2}{|l|}{ Meaning } \\
\hline$E_{j}$ & \multicolumn{2}{|l|}{ Equilibrium points $j$} \\
\hline$S^{*}$ & \multicolumn{2}{|c|}{ Equilibrium point value for $S$} \\
\hline$S_{i}^{*}$ & \multicolumn{2}{|c|}{ Equilibrium point value for $S_{i}$} \\
\hline$I^{*}$ & \multicolumn{2}{|c|}{ Equilibrium point value for $I$} \\
\hline$I_{i}^{*}$ & \multicolumn{2}{|c|}{ Equilibrium point value for $I_{i}$} \\
\hline$N^{*}$ & \multicolumn{2}{|c|}{ Equilibrium point value for $N$} \\
\hline$N_{i}^{*}$ & \multicolumn{2}{|c|}{ Equilibrium point value for $N_{i}$} \\
\hline$C_{0}$ & \multicolumn{2}{|l|}{ Consumption number } \\
\hline$C_{i}$ & \multicolumn{2}{|c|}{ Consumption number associated with resource $i$} \\
\hline $\bar{C}_{0}$ & \multicolumn{2}{|c|}{ Consumption number for $n>1$} \\
\hline$R_{0}$ & \multicolumn{2}{|l|}{ Reproduction number } \\
\hline $\bar{R}_{0}$ & \multicolumn{2}{|c|}{ Reproduction number for $n>1$} \\
\hline$\lambda_{j}$ & \multicolumn{2}{|l|}{ Eigenvalue $j$} \\
\hline
\end{tabular}

Symbols that do not appear in this list are used only to simplify the writing of equations and are defined in the text. 